\title{
A methachromatic-based experimental model for identification of bowel as the focus of an acute inflammation*
}

\author{
Sravya Sowdamini Nakka ${ }^{1}$, Jessica Johansson ${ }^{2}$, Faisal Shahzad ${ }^{1}$, Anders Hanning ${ }^{3}$, Fariba Nayeri ${ }^{1,4 \#}$ \\ ${ }^{1} \mathrm{R} \&$ D Department, Institution for Protein Environmental Affinity Surveys, PEAS Institut, Linköping, Sweden \\ ${ }^{2}$ Department of Clinical Medicine, School of Health and Medical Sciences, Örebro University, Örebro, Sweden \\ ${ }^{3}$ Episentec AB, Fjällvägen 6B, Sollentuna, Sweden \\ ${ }^{4}$ Division of Infectious Diseases, Department of Clinical and Experimental Medicine, Linköping University Hospital, Linköping, Sweden \\ Email: " Fariba.Nayeri@lio.se
}

Received 27 December 2012; revised 25 January 2013; accepted 2 February 2013

\begin{abstract}
Diarrhea is the most common symptom of acute inflammation in gastrointestinal tract and the patients are isolated in order to inhibit transmission and to conduct investigations. Yet there is no standard test to distinguish gastrointestinal infection from more generalized diseases at admittance which might cause delay in therapy. Hepatocyte growth factor (HGF) is produced upon injury by mesenchymal cells. On the contrary to chronic inflammation, HGF produced in the course of acute inflammation is biologically active and shows binding affinity to heparan sulphate proteoglycan (HSPG) and dextran sulphate (DS). Based on this phenomenon, an agarose gel containing DS was prepared and immobilized on loops to investigate the feces samples for the presence or absence of growth factors such as HGF with affinity to DS. The study is conducted as a clinical evaluation of an experimental model to distinguish acute infectious gastroenteritis from other causes of diarrhea. 656 fecal samples gathered consequently from patients seeking for bowel disturbances and healthy were tested by the test and the medical reports were investigated. Upon interaction with DS, methylene blue changes color to pink. This phenomenon was inhibited by HGF and converted by addition of anti-HGF antibodies to the samples. The test distinguished acute infectious gastroenteritis with high sensitivity and specificity $(96 \%$ and $92 \%$ respectively) from other causes of diarrhea. We introduce a metachromatic experimental model that might distinguish acute inflammation in alimen-
\end{abstract}

\footnotetext{
"There is a pending patent regarding the gel preparation.

The project was funded by ALF Grants County Council Östergötland and PEAS Institut.

The study was approved by the Ethical Committee in Linköping (M151-09)

${ }^{\#}$ Corresponding author.
}

tary tract from other causes of diarrhea. This model might be used in developing rapid diagnostic tests.

Keywords: Gastroenteritis; Hepatocyte Growth Factor; HSPG; Metachromasy; Rapid Test

\section{INTRODUCTION}

Besides to acute infectious gastroenteritis, diarrhea is the symptom of several other serious systemic infections as well as inflammatory diseases that need immediate intervention. Because the therapeutic strategies differ significantly between different diseases it is of great value to establish the right diagnosis as soon as possible. The most available procedures that are undertaken to establish diagnosis in patients with diarrhea by examination of stool are direct microscopy, cultures, parasitological examinations, PCR and Elisa tests to identify virus, determination of hemoglobulin, calprotectin and toxins. However, with respect to antibiotic consumptions and/or low antigen burden the tests have a limited sensitivity $[1,2]$.

Studied widely since 20 years ago hepatocyte growth factor (HGF) has shown to be highly involved in biological procedures relating to development and regeneration $[3,4]$. Thus changes in presence, structure and configuration of this growth factor might have consequences in lack of healing or disease development [3]. Biosensor technology, such as surface plasmon resonance (SPR), is a reproducible method for real-time evaluation of binding affinity to ligands and receptors [5]. Biologically active HGF shows binding affinity to HSPG in SPR system and the affinity is decreased after addition of sulphated oligosaccharides such as DS to the samples $[6,7]$. Biosensor coated with DS was recently introduced for detection of HGF in cellular medium [8]. HGF as a potential therapeutic strategy in treatment of chronic inflammatory bowel diseases has been discussed in recent 
studies [9-11]. However it might be of importance to further study the presence and quality of HGF during different inflammatory processes since high amounts of HGF is produced during acute inflammation in bowel [12].

Metachromasy is a characteristic color change that certain aniline dyes exhibit when bound to chromotrope substances [13]. This phenomenon has been widely used in study of tissue sections. Methylene blue (O-Toluidine) is considered an excellent metachromatic dye, which upon binding to high molecular weight polysaccharides, such as DS, changes the color of the indicator solution from blue to pink [13].

Taking advantage of previous studies and observations, an experimental model has been established and presented using agarose membrane containing DS sodium salt that changes color to pink in contact with methylene blue $[7,8]$. Incubation of this membrane in feces in 10 seconds might distinguish samples containing active HGF that binds to DS and inhibits metachromasy.

\section{MATERIALS AND METHODS}

DS sodium salt from Leuconostoc spp. with molecular weight 6500- > 500,000, high resolution agarose, Toluidine Blue $\mathrm{O}$ (trade name for Methylene Blue indicator solution), Fetal bovine serum (FBS), Proclin 300, D-(+)Glucose were all obtained from Sigma Aldrich, Sweden. Mannitol (150 mg/mL) was from Fresenius Kabi AB, Sweden, Phosphate Buffer Solution (pH 7.4) from Apoteket, Linköping, Sweden, Recombinant HGF from R \& D systems Inc., Minneapolis, USA and Styrene plastic loop $1 \mu \mathrm{l}$ and micro-tubes Safe Seal $1.5 \mathrm{~mL}$ were obtained from Sarstedt AB, Linköping, Sweden.

\subsection{Preparation of Gel and Immobilisation on Loops (the Test)}

DS MW 100,000 (0.9 mg), Agarose high resolution (9 mg), Glucose (4 mg), $300 \mu \mathrm{l}$ PBS in $700 \mu \mathrm{l}$ distilled water and Mannitol $(50 \mu \mathrm{l})$, were mixed and heated until a clear solution. Plastic loops were dipped once in the solution, approximately $5 \mathrm{~mm}$ above the loop, and were next placed with the loop pointing upwards to dry at

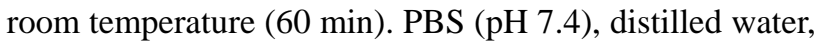
$0.5 \%$ serum albumin solution or $0.5 \%$ FBS were used as negative controls. Recombinant HGF (R \& D) diluted in 0.5\% FBS (125 - $8000 \mathrm{pg} / \mathrm{ml}$ ) was used as positive control.

\subsection{Preparation of Indicator and Feces Diluent}

A $1 \%$ methylene blue (100 mg in $10 \mathrm{ml}$ distilled water) stock solution diluted to $0.003 \%$ (1 mL in $300 \mathrm{ml}$ distilled water) was used as indicator solution. Proclin 300 (50 $\mu \mathrm{l}$ in $10 \mathrm{ml}$ ) was added to FBS which was used as feces dilution solution.

\subsection{Participants}

\subsubsection{Retrospective}

Feces samples that were gathered in 2003 during a previous study of HGF in blood and feces of patients with IBD and infectious gastroenteritis $(n=146)$ were rethawed once and examined by the test. This material is consisted of healthy volunteers and patients with defined diagnosis (Inflammatory Bowel Disease; IBD n = 58, 19 - 80 years old and 32 women, Infectious gastroenteritis n = 70, 9 - 90 years old and 37 women and healthy $n=6$ in 3 consecutive occasions, 19 - 35 years old, 3 women).

\subsubsection{Prospective}

This material (1 - 100 years old) was collected at the Department of Infectious Diseases $(n=63$ January 2010-June 2011) or from samples sent by other wards to the Departments of Biochemistry ( $n=106$, 18th May1st June 2011) and Microbiology ( $\mathrm{n}=313$, 1st -30th June 2011). The samples were coded at once and unidentified. The project leader studied the patient-journals and the result of the test and the medical history were documented directly. The files were updated daily and the results of routine tests, $\mathrm{X}$-rays, endoscope procedures and final diagnosis before patient was dismissed were documented consequently. Total 482 feces samples from patients that searched the health care centers $(n=122)$ or were admitted to the University Hospital in Linköping, County hospitals in Norrkoping and Motala $(\mathrm{n}=360)$ were analyzed consecutively with the test within 48 hours after collection before the culture or PCR results were available. Further 27 culture negative feces samples from healthy volunteers without diarrhea (9 - 73, Median 53 years, 17 women) were included. The physician in charge in each ward conducted the diagnosis procedure and treatment. Therefore the reference tests were performed accordingly on patients and it was not known to the physicians which patients were going to be included in the study.

\subsection{Assay Accomplishment and Interpretation of Results}

Feces samples stored in room temperature for 15 minutes prior to performing the test. The loop was first immersed in feces for $10 \mathrm{sec}$, the excess feces was gently wiped off and the loop was next placed in micro tubes containing $70 \mu \mathrm{l}$ indicator solution for $10 \mathrm{sec}$ and twisted gently by rolling the loop between thumb and index finger for 1 sec. The loop was removed and the color observed. A blue color (positive test) indicates presence of active HGF in feces, and a pink color (negative test) indicates no presence or presence of HGF with no affinity to DS. In the case of a negative result $15 \mu \mathrm{l}$ feces was diluted in $80 \mu \mathrm{L}$ dilution solution and the above process was re- 
peated. This procedure was undertaken to avoid false negative results relating to hook or prozone effect (too high level of binding growth factor in the sample) [14]. In the present work all of the fresh samples were tested originally and after dilution. Positive test both in original and diluted feces indicated a high DS affinity in feces HGF. Positive test in original feces with diarrhea and negative in diluted one indicated a low affinity of HGF to immobilized DS. When the results were negative in original feces, but turned to positive after dilution, presence of HGF with high affinity to DS is suspected (positive). When the results are negative in both original and diluted feces it might show that no HGF with affinity to DS is present in feces. The CMYK system was used for interpretation of colors as follows: 701500, 502500, 453000 are ranges of positive result and 354000, 254000 as negative results [15].

\subsection{Test Reproducibility}

In order to analyze all the feces samples collected during study period it was necessary to prepare different batches of loops to supply the study with test material. Approximately 500 loops could be prepared from $1 \mathrm{ml}$ DS gel from each batch prepared. In order to control method variability in preparation, 5 - 10 samples were analyzed with; the old and the new batch of loops. In case the result of sample analysis differed completely in more than two samples, the complete loop batch was discarded and a new batch was prepared. Twenty loops from each batch were also tested with negative controls (PBS, Albumin, FBS, distilled water) and positive control $(125 \mathrm{pg} / \mathrm{ml}$ recombinant HGF). Positive and negative controls were also used prior to analysis of samples daily. Feces samples were analyzed in duplicates.

\subsection{Inhibition of Interaction}

Feces sample from 2 patients with diarrhea caused by Salmonella enteritis (27 ng/ml HGF) and Clostridium difficile (7.9 ng/ml HGF) were diluted in distilled water, stirred and centrifuged for 20 minutes at $3000 \mathrm{~g}$. The supernatant was diluted in distilled water 1:10 and sterile filtered. Anti-human HGF antibody $0.1 \mathrm{mg} / \mathrm{ml}$ (Sigma Aldrich) was added 1:5 to the samples and incubated in 1 h $37^{\circ} \mathrm{C}$. Distilled water was added $1: 5$ to the control sample. Forty loops (10 loops for each sample with and without antibody) were tested.

\subsection{Reference Tests}

Stool examinations by means of a combination of microscopic, virology (Calicivirus with PCR, Rotavirus and Adenovirus antigen detection with ELISA) and bacterial culture methods as well as serological and toxin-identi- fication techniques (Clostridium difficile toxin), method for detection of semi-quantitative calprotectin and fecal hemoglobulin were performed as routine tests at the University Hospital in Linköping. A variety of $\mathrm{X}$-ray and endoscopic techniques were utilized as indicated.

\section{Statistics}

Regression analysis was performed for correlations using Graphpad prism version 5. Specificity (number of true negative/number of true negative + number of false positive), Sensitivity (number of true positive/number of true positive + number of false negative), Positive Predictive Value (PPV = True positive/true positive + false positive), Negative Predictive Value $(\mathrm{NPV}=$ True negative/true negative + false negative) and Accuracy (True positive + true negative/true positive + true negative + false positive + false negative) of the test results were calculated. The Regression analysis was performed to calculate the correlation between parameters (Graphpad prisma version 5).

\section{RESULTS}

\subsection{Gel Preparation}

The first observation showing that addition of HGF to DS solution could inhibit the interaction and thereby changing of color of methylene blue, motivated the testing of HSPG and DS with different molecular weights and concentration with different diluents and compositions. DS MW 100,000 in the composition mentioned in Material \& Method gave the most reliable results.

\subsection{The Diagnostic Procedures}

The feces cultures $(\mathrm{n}=195)$, diagnostic virology tests ( $\mathrm{n}$ $=58)$, toxin $(\mathrm{n}=318)$, parasitological tests $(\mathrm{n}=44)$, semi-quantitative calprotectin $(\mathrm{n}=111)$ and feces hemoglobulin $(n=61)$ were performed on feces samples $(n=$ 482) at admittance. These tests were performed on the same feces samples used in this study. However the results of the test were observed and documented before the results of reference tests were available.

\subsection{Infectious Gastroenteritis}

After the results of routine tests were available it was shown that 137 cases ( 8 - 100 years, Median 70 years old, 61 women, loose feces $n=131$ ) the stool examinations revealed positive cultures (Salmonella $n=6$, Campylobacter $n=10)$, Clostridium difficile toxin $(n=89)$, viral diagnosis $(n=24$, Calicivirus $n=7$, Rotavirus $n=16$, Adenovirus $n=1)$ parasites $(n=8$, Giardia intestinalis $n$ $=5$, Schistosomia mansoni $\mathrm{n}=1$ and Blastocystis homonis $n=2$ ). Double infection was observed in 2 cases. Together 207 feces samples (2003 and 2011) had verified 
infection (Figure 1). The test was negative in 2 cases without diarrhea and 5 cases with diarrhea.

\subsection{Non-Infectious Diarrhea}

In 143 cases ( 3 - 96, median 55 years, 64 women, loose feces $n=95$ ) bowel as focus of infection was ruled out. However in one patient with diarrhea Blastocystis hominis cyst was found in feces but the performed X-ray and endoscopy showed terminal ileitis. In this group the test was positive in six cases (Table $\mathbf{1}$ ).

\subsection{Inflammatory Bowel Diseases (IBD)}

The patients with bowel disturbances in which infection could not be verified by stool examinations and debut or exacerbation of IBD was suspected, were classified under this sub-group. Total 24 samples from 21 patients (14 83, median 59.5 years, 14 women, loose feces $n=20$, ulcerous colitis $n=15$, crohn disease $n=4$, microscopic colitis $n=4$, celiac disease $n=1$ ) were collected. The diagnosis was verified by a recent endoscopy in 14 cases. Out of 15 samples of patients diagnosed as exacerbation of ulcerous colitis, the test was positive in both original and diluted samples in 4 patients (Table 2). Endoscopic examination $(n=4)$ revealed acute or exacerba- tion of colitis.

Fifty-eight samples from 2003 with defined IBD diagnosis were included in this group and in total 82 samples with IBD the test was positive in 14 cases (Table $\mathbf{1}$ ).

\subsection{Sepsis}

Diarrhea during the course of sepsis with multiple organ dysfunctions was observed in 22 samples from 19 patients (32 - 92, median 69 years, 15 women). The test was positive in 18 cases (Table 2). The blood cultures yielded growth of Staphylococcus aureus in 3 cases, Klebsiella pneumoniae in 2 cases, Klebsiella pneumoniae + Bacteroides fragilis in one case, Streptococcus pneumoniae in one case, Streptococcus Oralis in one case, Streptococcus agalactiae (GBS) + Candida albicans in one case, Proteus mirabilis in 1 case, Candida albicans in one case, Staphylococcus aureus + Bacteroides fragilis in one case, E. coli in 2 cases, Enterococcus gallinarum in one case and aerococcus spp. in one case. The blood cultures were negative in 3 cases with sepsis and multiple organ failure.

This group is excluded from statistical analysis because the patients had generalized inflammation and organ dysfunction.

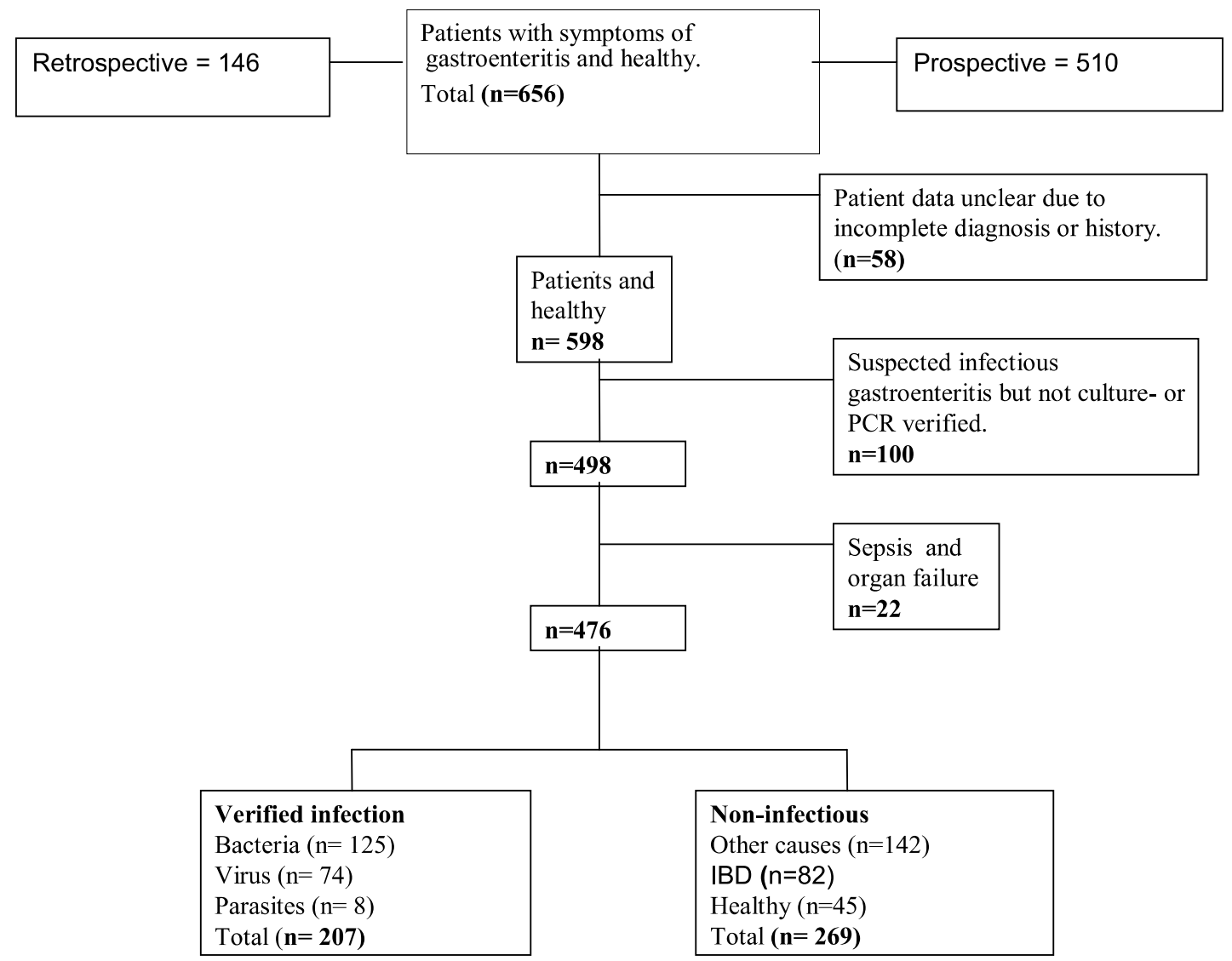

Figure 1. The study population presented in a flow chart. 
Table 1. Validation of the diagnostic test.

\begin{tabular}{cccccc}
\hline \multirow{2}{*}{ Diagnostic test result } & \multirow{2}{*}{ Verified infectious diarrhoea } & \multicolumn{3}{c}{ Non-infectious cases } & Total \\
\cline { 3 - 6 } & & Other causes & healthy & IBD \\
\hline Positive & 200 & 6 & 0 & 14 & 220 \\
Negative & 7 & 136 & 45 & 68 & 256 \\
Total & 207 & 142 & 45 & 82 & 476 \\
\hline
\end{tabular}

Sensitivity and specificity of the test were calculated as $96.6 \%$ and $92.5 \%$ respectively while Positive Predictive Value (PPV) and Negative Predictive Value are calculated as $90.9 \%$ and $97.2 \%$. Validation of diagnostic test results are found to be $94.3 \%$ accurate.

Table 2. The index test result in feces samples and subgroups collected in 2011.

\begin{tabular}{ccccc}
\hline Subgroups & Positive (positive) & Positive (negative) & Negative (positive) & Negative (negative) \\
\hline Sepsis & 13 & 4 & 1 & 4 \\
Verified infection & 102 & 13 & 17 & 5 \\
Non-infectious diarrhea & 3 & 3 & 0 & 136 \\
Suspected Infection & 71 & 8 & 15 & 6 \\
IBD & 4 & 5 & 0 & 15 \\
\hline
\end{tabular}

Positive/negative $=$ test positive in undiluted form, Negative/Positive $=$ test positive after dilution (hook effect). Negative/negative result is considered as negative test. Results mentioned in brackets represent the result obtained upon dilution of faeces samples in 1:5 ration of FBS-Proclain 300 solution.

\subsection{Suspected Infection}

In 100 patients ( 4 - 93, median 64 years, 61 women loose feces $n=95$ ) reference tests did not reveal infection but infectious gastroenteritis was highly suspected because of medical history and/or previous cultures. The test was positive in 94 cases (Table 2). This group is not included in statistical analysis because the diagnosis was not objectively verified.

\subsection{Unclear Cases}

The medical history and/or the diagnostic procedure were incomplete or classed as high secret and therefore it was not possible to find out the cause of diarrhea in 58 patients (29 women) which were excluded from statistical calculations (Figure 1).

\subsection{Estimates}

\section{1) Inhibition of HGF}

Incubation of diluted feces with anti HGF antibodies resulted in inhibition of $\mathrm{HGF}$ and a negative result in $80 \%$ (16 out of 20 loops).

2) Reliability of test

Sensitivity, specificity, positive predictive value and negative predictive value as well as accuracy were calculated in two groups; verified infectious gastroenteritis ( $n=207$ ) and non-infectious gastroenteritis which in turn included patients with IBD, patient with other systemic diseases and healthy $(n=268)$. As shown in Table 1 , the test could distinguish acute infectious gastroenteritis with a sensitivity of $96.6 \%$ and specificity $92.4 \%$ and a posi- tive predictive value of $90.9 \%$ and negative predictive value of $97.2 \%$. The accuracy of test was $94.3 \%$.

The results of the test did not correlate to fecal calprotectin $\left(\mathrm{R}=0.015, \mathrm{R}^{2}=0.02\right)$ or fecal hemoglobulin $(\mathrm{R}=$ $\left.0.29, \mathrm{R}^{2}=0.08\right)$ but correlated positively to loose feces $\left(\mathrm{R}=0.59, \mathrm{R}^{2}=0.35 \mathrm{p}<0.001\right)$. Fecal calprotectin had no correlation to feces consistence $\left(R=0.09, R^{2}=0.008\right)$ but correlated to fecal hemoglobulin $\left(R=0.47, R^{2}=0.22\right.$, $\mathrm{p}=0.001)$.

\section{DISCUSSION}

In the present work an experimental model for evaluation of HGF in faeces samples and determination of bowel as the focus of acute inflammation is introduced. The chromotropic character of HSPG and DS in inducing metachromatic colour change in methylene blue and toluidine blue has been used in development of test. We have shown that the results can discriminate an acute inflammation in bowel from a chronic one with high test predictive values.

Our observations regarding discrepancy between concentration of HGF (ELISA) and its binding affinity to ligands (SPR) together with decreased biological activity in vitro during chronic inflammation was the basis of development of a method to discriminate chronic injuries in which treatment with biologic active HGF might be beneficial [16-18]. Using the underlying theory a DS membrane (the test) was prepared to determine the binding affinity of HGF to DS in feces [19,20]. During preparation of the membrane it was shown that the molecular weight of DS was crucial in reaction and stability 
of the membrane. DS, 100,000 MW was used and this was in agreement to results reported by Berger et al., [8] Preparation of membrane was also dependent on volume, $\mathrm{pH}$ and temperature of solutions [13].

Diarrhea is the major symptom of food borne illnesses which in turn can be divided to bacterial, viral, chemical and parasitic categories. A large portion of the outbreaks are of unknown etiologies (45.1\%) [21] and therefore the limited tests used at the laboratory could not possibly rule out infection in all patients with gastroenteritis. In order to evaluate the sensitivity of routine diagnostic tests to determine the etiology of gastroenteritis in our ward we studied journal of patients that were admitted to the Department of Infectious Diseases in Linköping and were dismissed under diagnosis acute infectious gastroenteritis $(n=181$, January to December 2011). Feces cultures (at least once in all of the cases), viral diagnostic tests $(n=123)$, direct microscopy and parasitological tests $(n=51)$ were performed accordingly which could verify infection in $59 \%$ of cases (submitted manuscript).

The test recognized acute inflammation caused by infection with a high sensitivity in patients with diarrhea. When diarrhea was not the presenting symptom the test was negative in spite of positive cultures. It might indicate that the test is sensitive to the grade of inflammation not presence of an infectious agent such as in carriers. In the patients with sepsis and multiple organ failure and diarrhea, positive test might indicate a generalized inflammatory process and a higher risk for bacteremia.

Calprotectin (semi-quantitative and quantitative) tests are recently been used as a routine to rule out a chronic inflammatory process in bowel [22]. There was no significant correlation between the test and calprotectin results in our observation. However in cases with negative calprotectin and positive test viral and parasitic infections might be suspected. On the other hand positive calprotectin together with negative test might have values in detection of IBD (data not shown).

We conclude that Dextran-activity test is a simple experimental model for evaluation of HGF in feces. The results are in agreement with our previous observations and the model might be used to develop rapid and costeffective tests for distinguishing cases in which bowel is the focus of acute inflammation such as in food borne outbreaks in need of isolation. Special attention should be paid to cases of gastroenteritis with negative test which need complementary examinations to rule out more serious conditions in need of appropriate therapeutic strategies without delay.

\section{ACKNOWLEDGEMENTS}

We are grateful to Tayeb Nayeri, Prof. Fredrik Winqvist and Per Stalhandske for their valuable suggestions in the development of method.
We are also grateful to Clinical and Laboratory staff of Linköping University hospital in helping us with collection of samples.

\section{REFERENCES}

[1] Kirby, A. and Iturriza-Gómara, M. (2012) Norovirus diagnostics: Options, applications and interpretations. Expert Review of Anti-Infective Therapy, 10, 423-433. doi:10.1586/eri.12.21

[2] Holtz, L.R., Neill, M.A. and Tarr, P.I. (2009) Acute bloody diarrhea: A medical emergency for patients of all ages. Gastroenterology, 136, 1887-1898. doi:10.1053/j.gastro.2009.02.059

[3] Nakamura, T., Sakai, K. and Matsumoto, K. (2011) Hepatocyte growth factor twenty years on: Much more than a growth factor. Journal of Gastroenterology and Hepatology, 26, 188-202. doi:10.1111/j.1440-1746.2010.06549.x

[4] Doeppner, T.R., Kaltwasser, B., ElAli, A., Zechariah, A., Hermann, D.M. and Bahr, M. (2011) Acute hepatocyte growth factor treatment induces long-term neuroprotection and stroke recovery via mechanisms involving neural precursor cell proliferation and differentiation. Journal of Cerebral Blood Flow Metabolism, 31, 1251-1262. doi:10.1038/jcbfm.2010.211

[5] Liedberg, B., Nylander, C. and Lundstrom, I. (1995) Biosensing with surface plasmon resonance-how it all started. Journal of Biosensors and Bioelectronics, 10, $1-9$.

[6] Nayeri, F., Xu, J., Abdiu, A., Nayeri, T., Aili, D., Liedberg, B., et al. (2006) Autocrine production of biologically active hepatocyte growth factor (HGF) by injured human skin. Journal of Dermatological Sciences, 43, 4956. doi:10.1016/j.jdermsci.2006.03.004

[7] Nayeri, F., Nayeri, T., Aili, D., Brudin, L. and Liedberg, B. (2008) Clinical impact of real-time evaluation of the biological activity and degradation of hepatocyte growth factor. Journal of Growth Factors, 26, 163-171. doi:10.1080/08977190802128083

[8] Berger, M., Welle, A., Gottwald, E., Rapp, M. and Lange, K. (2010) Biosensors coated with sulfated polysaccharides for the detection of hepatocyte growth factor/scatter factor in cell culture medium. Journal of Biosensors and Bioelectronics, 26, 1706-1709.

doi:10.1016/j.bios.2010.07.065

[9] Yamaji, N., Ido, A., Moriuchi, A., Numata, M., Setoyama, H., Tamai, T., et al. (2011) Hepatocyte growth factor ameliorates mucosal injuries leading to inhibition of colon cancer development in mice. Oncology Reports, 26, 335-341.

[10] Linares, P.M. and Gisbert, J.P. (2011) Role of growth factors in the development of lymphangiogenesis driven by inflammatory bowel disease: A review. Journal of Inflammatory Bowel Diseases, 17, 1814-1821. doi:10.1002/ibd.21554

[11] Cammarota, R., Bertolini, V., Pennesi, G., Bucci, E.O., Gottardi, O., Garlanda, C., et al. (2010) The tumor microenvironment of colorectal cancer: Stromal TLR-4 expression as a potential prognostic marker. Journal of 
Translational Medicine, 8, 112-128. doi:10.1186/1479-5876-8-112

[12] Nayeri, F., Almer, S., Brudin, L., Nilsson, I., Akerlind, B. and Forsberg, P. (2003) High hepatocyte growth factor levels in faeces during acute infectious gastroenteritis. Scandinavian Journal of Infectious Diseases, 35, 858862. doi:10.1080/00365540310016484

[13] Bergeron, J.A. and Singer, M. (1958) Metachromasy: An experimental and theoretical reevaluation. Journal of Biophysical and Biochemical Cytology, 4, 433-457. doi:10.1083/jcb.4.4.433

[14] Fangous, M.S., Kerspern, H., Moineau, M.P., Kerlan, V., Alavi, Z. and Carre, J.L. (2012) The hook effect in calcitonin immunoradiometric assay: A case report. Annual Endocrinology Journal, 73, 552-555. doi:10.1016/j.ando.2012.07.681

[15] Pham, N.A., Morrison, A., Schwock, J., Aviel-Ronen, S., Iakovlev, V., Tsao, M.S., et al. (2007) Quantitative image analysis of immunohistochemical stains using a CMYK color model. Journal of Diagnostic Pathology, 2, 8-17. doi:10.1186/1746-1596-2-8

[16] Lönn, J., Starkhammar, J.C., Kälvegren, H., Brudin, L., Skoglund, C., Garvin, P., et al. (2012) Hepatocyte growth factor in patients with coronary artery disease and its relation to periodontal condition. Results in Immunology, 2, 7-12. doi:10.1016/j.rinim.2011.12.002

[17] Lönn, J., Shahzad, F., Uhlin, F., Bengtsson, T., Almroth,
G. and Nayeri, F. (2012) High concentration but low biological activity of hepatocyte growth factor in patients with chronic renal failure. Advances in Bioscience and Biotechnology, 3, 516-523. doi:10.4236/abb.2012.324068

[18] Lonn, J., Almroth, G., Brudin, L. and Nayeri, F. (2012) An antithrombin III product containing biologically active hepatocyte growth factor may be beneficial in deep ulcer infections. Cytokine, 60, 478-486. doi:10.1016/j.cyto.2012.05.023

[19] Nayeri, F., Nilsson, I., Brudin, L. and Almer, S. (2004) Stability of faecal hepatocyte growth factor determination. Scandinavian Journal of Clinical and Laboratory Investigations, 64, 589-597. doi:10.1080/00365510410002850

[20] Nayeri, F., Aili, D., Nayeri, T., Xu, J., Almer, S., Lundstrom, I., et al. (2005) Hepatocyte growth factor (HGF) in fecal samples: Rapid detection by surface plasmon resonance. BMC Journal of Gastroenterolology, 5, 13-20. doi:10.1186/1471-230X-5-13

[21] Bean, N.H., Griffin, P.M., Goulding, J.S. and Ivey, C.B. (1990) Foodborne disease outbreaks, 5-year summary, 1983-1987. MMWR CDC Surveillance Summaries, 39, 15-57.

[22] Kostakis, I.D., Cholidou, K.G., Vaiopoulos, A.G., Vlachos, I.S., Perrea, D. and Vaos, G. (2012) Fecal calprotectin in pediatric inflammatory bowel disease: A systematic review. Journal of Digestive Diseases and Sciences, in Press. doi:10.1007/s10620-012-2347-5 